\title{
Regionalization and Health Networks
}

Ana Luiza d'Ávila Viana ${ }^{1}$ Aylene Bousquat ${ }^{1}$ Guilherme Arantes Melo ${ }^{2}$ Armando De Negri Filho ${ }^{3}$ Maria Guadalupe Medina ${ }^{4}$
${ }^{1}$ Faculdade de Saúde Pública, Universidade de São Paulo. Av. Dr. Arnaldo 715, Cerqueira César. 01246-904. São Paulo SP Brasil.

aylenebousquat@usp.br ${ }^{2}$ Universidade Federal de São Paulo. São Paulo SP Brasil.

${ }^{3}$ Laboratório de Inovação, Hospital do Coração. São Paulo SP Brasil.

${ }^{4}$ Instituto de Saúde Coletiva, Universidade Federal da Bahia. Salvador BA Brasil.

\begin{abstract}
The aim of this article was to reflect on the challenges faced in building the Health Regions and Networks in Brazil. These reflections result from studies conducted in several health regions. The central challenges for the constitution of the Health Regions and Networks are as follows: the Care Model, Primary Health Care, Care for Users with Chronic Diseases, and Hospital Care. In order to consolidate the regions and networks the organization needs to incorporate an ethical sense: focusing on social, public and individual needs, derived from comprehensive care, human and social rights, in broad, multi-scale and inter-sectorial interactions, with the constitution of a systemic, regionalized innovation. Of prime importance is the capacity to devise which general principles are responsible for maintaining the unity (mode of care), seeking to outline the coherence of the different provision models that the system might develop according to the social needs in regional settings. Without that, users will continue to seek and create ways of accessing health services that challenge the rationale of those very services. Hospital reform is also required to integrate and reorient the hospital networks. These reflections are important for the SUS to uphold its solidarity project, expressed in the trinity of universality-comprehensiveness-equality.
\end{abstract}

Key words Regions and networks, Comprehensive care, Regionalization of health services 


\section{Introduction}

The organization of Brazil's Unified Health System (Sistema Único de Saúde - SUS) may be divided into two political cycles. The first (1988 to 2000) focused on decentralization to subnational entities, with local government playing an important role in this cycle. The second cycle (2000 to present) prioritized the creation of Health Regions, a process otherwise known as regionalization, and Health Care Networks (Redes de Atenção à Saúde - RAS). These periods differed from each other in the following aspects: political, economic and social context; healthcare models; funding arrangements; type of decentralization; key stakeholders; base of political support; "territorialization"; and management and regulation models ${ }^{1}$.

The Brazilian Constitution provides that the health service delivery shall be organized through networks aimed at building integrated systems of healthcare, these are known in different countries and international organizations as integrated service networks, regional networks, integrated services, and integrated care.

These networks have become bastions of a new approach to health service management and organization and facilitators of the integration of systems on a territorial basis. Guided by federal regulations, this process began in 2000 with the purpose of regionalizing the health system, continuing 10 years later with the introduction of RAS as a mechanism for building integrated health systems ${ }^{1}$.

It is no easy task to understand how the decentralization process deviated from regionalization in the first cycle or explain why regionalization initiatives lag behind network initiatives. It is equally difficult to characterize how, in the second cycle, regionalization changed the face of decentralization, making it less fiscal and more administrative, while at the same time maintaining shared management by government entities and between service managers and providers, but without effective public participation ${ }^{2}$.

This task requires a line of research that encompasses both the institutional aspects of this process, such as culture and values, and historical aspects involving the multiple bases of the constitution of the SUS. Moreover, any analysis of the second political cycle requires new tools that were not part of the theoretical framework adopted to assess the first cycle, such as multi-scale analysis, which provides a greater understanding of the RAS and urban complexity resulting from the large degree of interdependence between urban, peri-urban and rural spaces ${ }^{1,2}$.

Similarly, the transformation towards integrated care requires an integrated vision of levels of care that goes beyond the closed approach to complexity, which recognizes that care involves a combination of services provided by different providers and professionals and different types of interaction with the population. In other words, it is the patient's needs that should determine the urgency of the case and the timing, severity and complexity of the response, whereby the assessment of performance is centered on health outcomes. However, this dimension is rarely assessed, meaning that the performance of the RAS and the SUS is often left unquestioned.

The above is central to any analysis of the regionalization process and creation of the RAS in Brazil. However, it is necessary to consider other elements among which the following may be highlighted: a) an integrated vision of health systems and social determinants of health, with different types of intersectoral interventions coordinated by multiple agents and institutions; $\mathrm{b}$ ) definition of public-public and public-private contractual standards, given the rapid process of "entrepreneurization" public health services; c) a shift towards a new technological paradigm given that connectivity, integration and regulation require the use of a new technological framework with the widespread use of devices and platforms such as Telessaúde; d) new and complex contours of regulation, characterized by the classical regulation of access, professionals and providers (services), and care and assistance, which require new instruments that improve the regulation of care quality and safety ${ }^{1,2}$.

In light of the above, this article reflects upon the second cycle, focusing on the development of the health regions and networks. These reflections are the fruit of methodological strategies and the results of research conducted in various health regions across Brazil under the study Região e Redes (region and networks) ${ }^{1,2}$. We emphasize four central challenges facing the development of health regions and networks: the care model, primary health care (PHC), chronic care, and hospital care.

\section{Once Model or Multiple Models of Care for the RAS?}

The Brazilian health reform was underpinned by a common sense of multiple 'care models' working in parallel. The main systems of thought 
that kindled this debate have been reviewed by Paim $^{3}$. The dichotomy between curative medical care in the social security sphere and preventive care in the public health domain began in the 1930 s and continued up to the 1980s, when the 'comprehensiveness of curative and preventive actions' were embodied in the creation of the SUS'.

The idea of care approaches and models of service delivery gives rise to two well-defined dimensions: the systemic model of service organization/integration and the care delivery model ${ }^{3}$. In both situations, sound analysis reveals a clash between the 'time of the mentalities' perspective (practices/common technical sense or the 'sociology of absences' according to Boaventura Santos ${ }^{5}$ ) and 'time of the ideas' perspective (vanguard/frontiers or 'the sociology of emergences', revisiting the concept of nocht nicht, or 'not yet', developed by Ernst Bloch ${ }^{6}$ where emergence is seen as a new systemic balance, fruit of learning in complex adaptive systems).

We begin by comparing the classical vision of levels of care and the current perspective underpinning the systemic model in the regionalized RAS founded in different socioeconomic, cultural and territorial realities. The Family Health Program (FHP), proposed as a care model by the Basic Operational Norm 96, clearly embodies the ideal of organizing the system into levels of complexity. The belief that improving the referral and counter-referral process would contribute to overcoming fragmentation of the system and claiming that 'first it is necessary to strengthen primary care' are two common arguments in the field of research that illustrate technical common sense. Research has shown that the implicit conviction that strengthening the FHP model and the integration of specialized services into primary care would lead to a general remodeling of the system was not well founded. It is worth highlighting that referral between different levels of care is a common element of various health systems and that a recent meta-analysis of this problem concluded that systemic solutions are the only effective alternative ${ }^{7}$.

This is not to say that the idea of levels of complexity should be discarded, but rather that this model is outdated and is only able to offer piecemeal solutions. The enhancement of the quality of services across levels of care and of the integration between these services represents making up for slow progress rather than systemic regionalized innovation.

The organization of the health system into complex integrated networks is hampered by im- mature theoretical models. This will be an urgent task, both in theoretical and practical terms, in coming years.

It is important to clarify certain assumptions. Firstly, it is not a question of the everyday semantics of service 'networks' found decades ago in the literature - the same could also be said about the concept of 'system' and regionalization itself - but rather of a concept of complex RAS defined as complex adaptive systems with capacity for self-regulation built around an ethical sense of systemic organization. These systems are centered on individual and collective social needs derived from the integrality of human and social rights, promoting well-being through broad multi-scale, inter-sectoral, and often cross-sectoral, interactions. Understanding and planning complexity requires innovative tools such as network analysis and mathematical modeling that encompass consumer habits and expectations and service delivery capacity, with outcomes that can be measured by the public.

In this scenario, one of the consequences for the SUS culture is that PHC loses its status as a 'solution' and starts to be viewed as part of the problem, remembering that robust PHC is only possible through a health system. Various factors come into play in this aspect. PHC is a concept that developed in the 1970s, before the emergence of the still mechanical systems view into which it had to fit in. Furthermore, it has been organized on a municipal scale, which faces new challenges on a regional scale. In Canada, an altogether different reality, an "extremely difficult to reform" PHC was identified as a barrier to the regionalization process $^{8}$.

International experience shows that comprehensive systems are made up of varying health care delivery models ${ }^{9}$, which involve different strategies for enhancing efficiency and effectiveness ${ }^{10}$. This is particularly relevant here, given that the diverse realities across the country mean that it has not been possible to define a single watertight PHC model by decree. Plainly divided between the 'traditional model' and Family Health Strategy, reality imprints an array of models of PHC in practice. Whatever the model, by defining the type of access and supply of services for the patient, each have reached their historical limits. By contrast, in health reforms in other countries it has been recognized that a patient-centered approach is central to ensuring the efficiency and effectiveness of PHC and health systems themselves.

It is clear that a self-regulating, patient-centered, complex adaptive system - born out of 
various cultures - requires more than a single, rigid model. As Paim ${ }^{3}$ concludes: "From this perspective, it does not makes much sense to talk about a single care model for the SUS, given the heterogeneous nature of the reality of health in different regions, cities and territories across the country". The secret lies in the capacity to discern what general principals should guide service provision, while seeking to ensure a coherent set of care models designed to meet the social needs of different regional contexts.

The organization of preferential RAS as coordinated provider networks projected into service and care lines would appear to be a good starting point for developing a new model centered on the organization of systems and responses to this set of social needs. Clearly, however, given their current number, preferential RAS (the Stork Network, and the Urgent and Emergency Care Network, etc.) are a far cry from a complex network. Furthermore, these RAS face huge bureaucratic and normative challenges and it is difficult to see how it will be possible to shape a system around common aims without the meaningful participation of all stakeholders. This is one of the conclusions of an extensive review of large-system transformation in health care ${ }^{7}$, particularly in relation to the inclusion of doctors and service users in the decision-making process. Without the inclusion of state and private providers and key health professionals, especially doctors, the governance capacity of the SUS remains questionable.

The basic question remains: in which domain should the essential pillar of a systemic redefinition of this level of complexity be erected? Two arguments appear to be particularly relevant to answering this question. In the SUS, the indissoluble universality-comprehensiveness-equality triad is complemented by guaranteed access, which deconstructs the primacy of cost-effectiveness from which the discussion of networks in the US healthcare system ${ }^{11}$ originates, which demands greater regional planning capacity. The consistency between health services organization flows and the reality of regional geographic flows (cultural, economic, etc.) is another huge challenge.

A second rift between the time of mentalities (certainties) and time of ideas (guesses) with regard to models of health care is expected in the clinical model. Accustomed to operating across levels of complexity, clinical practice should be reorganized to meet the new requirements of the RAS model in which tackling fragmentation of care and, therefore, the continuity, integration and coordination of care is a central element. The challenge is therefore not replacing an acute care model with a chronic care model, but rather providing an adequate response to the needs, whatever their nature, expressed through citizenship, which differ considerably given heterogeneity across the country. Furthermore, these differences should be supported through the protection of the right to health, contributing towards the reduction of the huge inequalities that exist in our country.

\section{Challenges in the care of patients with chronic conditions in health regions and networks}

One of the many challenges facing the SUS has been and continues to be transforming an insufficient health services network, oriented towards acute and maternal and child care, into a health system capable of tackling the new epidemiological and demographic situation facing Brazilian society. With respect the care of patients with chronic conditions, progress has been made resulting from a number of factors, including the significant expansion of PHC services, enhanced access, greater availability of a regular sought of healthcare services, and pharmaceutical policies. Studies have shown that such measures have had the following impacts: reduction in cardiovascular and cerebrovascular mortality ${ }^{12}$; reduction in racial inequalities in hospital admissions for these conditions ${ }^{13}$; and a general reduction in inequalities in access to health services ${ }^{14}$, among others.

However, this progress has not been sufficient to ensure the provision of comprehensive care to these service users, who need frequent access to various points in the care network, are treated by various types of professionals, benefit from continuing health prevention and promotion actions, and develop self-care skills across their life-course. It is also worth highlighting that this problem is not exclusive to the Brazilian health system, as a plethora of studies show ${ }^{15,16}$.

Organization of RAS has been highlighted by the literature and multilateral organizations such as the Pan American Health Organization as a way of coordinating actions and services and potentially reducing the fragmentation of care, which is essential in the care of patients with chronic conditions, particularly for those suffering from more than one condition ${ }^{16,17}$. However, as Paim ${ }^{3}$ claims, it is necessary to clarify this debate, given 
that the RAS created in universal health systems are built on important clinical care coordination mechanisms, protocols, and articulation between professionals across various levels of care, while those set up in market systems are centered on competition. Thus, one of the pillars of an effective RAS is effective coordination of care. In other words, PHC should be robust and capable of coordinating the navigation of service users through different points of entry, ensuring care is provided at the right place and right time. Given the economic context and population size of the majority of Brazilian municipalities, it is impossible to provide this type of service without regional scale. It is also important to mention the major presence of the private sector, including both profit and non-profit organizations, and groups that take advantage of the privatization of healthcare, which sell services to the SUS across the country, particularly diagnostic services. Their market logic and frequent monopoly make it difficult for the majority of local governments to compete on their own.

The implementation of the RAS and care of patients with chronic conditions Network (Rede de Cuidado aos Pacientes com Doenças Crônicas) is still in progress and several studies conducted in various localities across the country have reported both progress and obstacles. Important steps forward include the enhancement of care coordination through the expansion of the coverage of PHC and specialized medical services, improvements made to the service procurement and regulation system, and the development of clinical and information coordination instruments ${ }^{18-20}$. On the other hand, evidence shows that the passage of service users through the system does not respect the agreements and norms established during the planning of health action and services $^{21,22}$. Furthermore, in the health regions, the navigation of service users tends to branch out across various services, leading to inappropriate or inefficient management of the patient referral process as services become more fragmented. In short, patients seek and create ways of accessing services that challenge the rationality of the health services, or in other words, they articulate flows in order to survive or ensure that they meet their real or symbolic demands.

The Região e Redes study compared managers' perceptions of the role played by $\mathrm{PHC}$ in the the care of patients with chronic conditions with the paths of patients with systemic arterial hypertension $(\mathrm{SAH})$ who had suffered a stroke in order to identify constraints and potentialities of the care process across various health regions. Semi-structured interviews were conducted with the managers and therapeutic itinerary mapping (TI) was used for patients. The latter is a particularly useful tool for analyzing health regions and networks ${ }^{21,22}$.

The results showed a vision of two very distinct and rarely converging worlds: that of managers and that of services users. Under the vision of managers, although there was a consensus that PHC should be the point of entry to the system, there was a general recognition of barriers to effective implementation, including the following: the need to provide more timely care; lack of general practice doctors; access difficulties at primary care centers (especially with respect to opening hours); limited resolvability capacity; and intermittent medicine dispensing. However, what really stands out in the interviews is that managers reproduce the line of arguments underlying the guidelines for the implementation of the national 'PHC policy, as if their idea was enough to ensure the effective implementation of this policy. Furthermore, they confirmed that $\mathrm{SAH}$ patient care was adequate in the majority of cases, event after the occurrence of strokes in primary care services.

On the other hand, the TIs showed that the majority of patients do not recognize that primary care services provide adequate care and are the main point of entry for accessing other health resources. PHC continues to be viewed predominantly as a locus for seeking and accessing simple procedures, such as medication and blood pressure measurement. Health promotion and prevention was shown to be secondary and the linkage between these actions and $\mathrm{PHC}$ was not clear. Furthermore, according to the interviewees, it is the patients themselves and/or their families who navigate their way through the system.

The results also revealed a public-private mix in the provision of health services in all regions, with direct payment of computed tomography exams, rehabilitation, consultations with specialist, among others. These services were actioned as a way of overcoming shortcomings/delays in the SUS. It is important to stress that this situation was not identified by the managers, who viewed services users exclusively as SUS patients.

With regard to strokes, access to the Mobile Emergency Care Service (SAMU) varied considerably across regions. In some cases it was exemplary while in others it was clearly deficient, illustrating regional differences in the implementation of this national policy. The same can be said 
for referral for inpatient admission. Although in the majority of regions patients were generally directed straight to the service which best meets their health needs, in a number of cases in the Costa Doce/Carbonífera Region the State of Rio Grande do Sul patients experienced difficulties in accessing the most appropriate service in a timely and efficient manner.

Lack of integration and formal mechanisms to promote integration between services and professionals was a recurring theme in the patient interviewees and was also mentioned by some managers. The results illustrate a high degree of fragmentation across the system and a virtual lack of regionalized networks in the cases under question. Although it is recognized that the expansion of PHC services has had a very positive impact on the care of patients with chronic conditions, it is evident that without effective coordination between the expansion and regionalization process and the creation of integrated networks these efforts are not sufficient to ensure that patients receive the necessary care. This points to the need to dissolve the prevailing dichotomy in this country, where the preventive or "preventivist" approach identified in the common sense of PHC is counterposed against a diagnostic/treatment/rehabilitation process strongly anchored in specialized in-patient care and out-patient services. One way of overcoming this dichotomy would be to rekindle the promotion of quality of life and health as a cross sectoral policy and care approach that tackles the social determinants of health, exposure to risks, and hazards and their individual and collective consequences, harnessing the potential of health promotion as a strategy for the integrating preventive, education, diagnostic, treatment and rehabilitation dimensions of RAS and regional and state systems and defining coherent models of healthcare provision.

\section{The role of hospital care in health regions and networks}

Another dichotomy that must be overcome is the quasi opposition between PHC and hospital care, in which the critical discourse regarding hospital-centrism has led to the abandonment of the latter as an object of strategic reflection on the SUS. Within this strategic debate, hospital care has been marginalized without any adequate assessment of structural deficiencies and systemic effectiveness and, therefore, without promoting a hospital care reform that integrates and re- orients these facilities towards the RAS in order to enhance the performance of PHC, including long term outpatient care, urgent and inpatient care.

It is important to mention that the marginalization of the debate about hospital care has taken place within a context of a continued reduction in hospital beds and an increase in the number of hospitals in the country. The number of hospital beds per 1,000 people overall and in the SUS currently stands at less than 2.1 and 1.5 , respectively ${ }^{23}$, representing a hug gap between Brazil and the universal health systems of OCDE member countries, which have at least 3 beds per 1,000 people $^{24}$. Furthermore, asymmetric patient flows concentrate demand in $25 \%$ of saturated permanent beds and $70 \%$ of beds have poor occupancy rates, resulting in an effective rate of 0.8 beds per 1,000 people and major regional differences in supply. This results in long waiting times for admission, both via urgent services and elective admission (given that $75 \%$ of admissions take place through urgent services), leading to increased mortality, complications and cumulative per patient healthcare costs ${ }^{23}$.

A review of this situation conducted in 2004 resulted in a proposal for a SUS hospital reform program and the creation of the Nacional Hospital Care Policy (PNHOSP, acronym in Portuguese) in 2013. However, despite the recommendations of the guiding document this has not led to the adoption of its systemic approach ${ }^{25}$.

Between 2003 and 2004, the debate surrounding hospital care in SUS was marked by a sense of crisis, characterizing policy orientations for the sector as fragmented and unspecific. Efforts were therefore directed at promoting reforms to the hospital care system to address lack of capacity and the failure of hospital services to meet health needs. This situation was aggravated by a lack of articulation between hospitals across the rest of the care network and the concentration of facilities in medium and large cities in the South and Southeast. A Ministry of Health report on the reform of Brazil's hospital care system ${ }^{26}$ highlights six key dimensions of the crisis and ways of dealing with them: financial, political, organizational, care, teaching and research, and social.

Despite of this systemic analysis, the federal government adopted short-term measures to address immediate problems of underfunding of small hospitals, teaching hospitals, and philanthropic hospitals and incentives for hospitals from the Stork Network and Urgent and Emergency Care Network, without promoting inte- 
gration between these hospitals and with PHC services and specialized outpatient services ${ }^{23}$.

Attempts to organize the hospital care policy and develop a framework for integrating this policy with the RAS led to the creation of the PNHOSP, which in 2013 also renounced the systemic approach resulting in guidelines that so far lack coordinated implementation ${ }^{23}$.

However, a number of successful experiences have been implemented. For example, using the strategic management approach Démarche Estrategique developed by Cremadez ${ }^{27}$ together with the broader complex adaptive systems framework $^{7}$, four states have made progress in including hospital care centers in lines of care and situating hospitals within regional care networks and systems, deconstructing the concept of hospitals as a single isolated unit and turning them into a "condominium" of services at different levels of care across the regional and state systems ${ }^{28}$.

These experiences clearly show that gains in hospital effectiveness and efficiency can only be achieved through organic integration into PHC and that effective PHC can only be achieved through systemic integration with hospitals and specialized care.

It is also clear that, within this systemic approach, state governments need to take the lead in organizing the supply of specialized and hospital care at regional and state level, with local governments leading the organization of PHC on a regional scale. In this respect, it falls on states to unify and promote democratic governance of planning and integrated regulation and procurement at regional level. This in turn requires the effective decentralization of technical capacities and political decision-making to regions and the consolidation of integrated hospital center management through the unification of procurement processes in the state-run network (direct administration, indirect administration, state and private sector providers) in order to achieve the effective integration of hospital services into the systemic design of the RAS and lines of care, while at the same time promoting spaces for the meaningful participation of providers and key professionals.

\section{Final Considerations}

Two key challenges stand out in the process of creating health regions and networks: the first refers to the need for states to exercise institutional leadership, while the second involves the need to engage health professionals (particularly doctors) as agents of change, alongside the incentive of social participation in health policies.

The second political cycle of the organization of the SUS did not have the political thrust of the first, precisely because it did not create a base of political support with sufficient strength to redefine actions, engage stakeholders and incentivize different types of institutional partnerships.

Though much has yet to be written about the challenges, solutions and impasses of this new cycle, but its shortcomings are sufficiently clear to demand more effective policies, new institutional environments geared towards cooperation and new participatory arrangements from all those responsible, without which it is likely that the tasks we have outlined above will not be able to be accomplished.

\section{Collaborations}

ALD’Á Viana, A Bousquat, GA Melo, A De Negri Fiho and MG Medina worked on the conception, research and final writing of the article. 


\section{References}

1. Viana AL, Ferreira MP, Cutrim MAB, Fusaro ER, Souza MR, Mourão L, Pereira APCM, Mota PHS, Iozzi FL, Uchimura LYT. O Processo de Regionalização no Brasil: influência das dimensões Política, Estrutura e Organização. Rev. bras. saúde matern. infant. 2017; 17(Supl. 1):S27-S43.

2. Viana AL, Bousquat A, Ferreira MP, Cutrim MAB, Uchimura LY, Fusaro ER, Souza MR, Mota PHS, Pereira APCM, Iozzi FL, Albuquerque MV. Região e Redes: abordagem multidimensional e multinível para análise do processo de regionalização da saúde no Brasil. Rev. bras. saúde matern. infant. 2017; 17(Supl. 1):S17-S26.

3. Paim JS. Modelos de Atenção à Saúde no Brasil. In: Giovanella L, Lobato LVC, Noronha JC, Carvalho AI, organizadores. Políticas e Sistema de Saúde no Brasil. 2a ed. Rio de Janeiro: Fiocruz; 2012. p. 459-491

4. Mello GA, Viana AL. Uma história de conceitos na saúde pública: integralidade, coordenação, descentralização, regionalização e universalidade. Hist. cienc. saúde -Manguinhos 2012; 19(4):1219-1239.

5. Santos BS. A Gramática do Tempo: para uma nova cultura política. São Paulo: Cortez; 2008.

6. Bloch E. O Princípio Esperança. Rio de Janeiro: Contraponto, Ed. UERJ; 2005-2006.

7. Best A, Greenhalgh T, Lewis S, Saul JE, Carrol S, Bitz J. Large-System transformation in health care: A realist review. The Milbank Quarterly 2012; 90(3):421-456.

8. Marchildon GP, Hutchison B. Primary care in Ontario, Canada: New proposals after 15 years of reform. Health Policy 2016; 120(7):732-738.

9. Pineault R, Levesque J-F, Roberge D, Hamel M, Lamarche P, Haggerty J. Accessibility and Continuity of Care: A Study of Primary Healthcare in Québec. Research report presented to the Canadian Institutes of Health Research and the Canadian Health Services Research Foundation. Montreal: Gouvernement du Québec et Centre de recherche de l'Hôpital Charles LeMoyne; 2009.

10. Martin LT, Plough A, Carman KG, Leviton L, Bogdan $\mathrm{O}$, Miller CE. Strengthening Integration of health services and Systems. Health Aff (Millwood) 2016; 35(11):1976-1981.

11. Kuschnir R, Chorny A. Redes de atenção à saúde: contextualizando o debate. Cien Saude Colet 2010; 15(5):2307-2316.

12. Rasella D, Harhay MO, Pamponet ML, Aquino R, Barreto ML. Impact of primary health care on mortality from heart and cerebrovascular diseases in Brazil: a nationwide analysis of longitudinal data. BMJ 2014; 349:g4014

13. Hone T, Rasella D, Barreto ML, Majeed A, Millett C. Association between expansion of primary healthcare and racial inequalities in mortality amenable to primary care in Brazil: A national longitudinal analysis. Tsai PLoS Medicine 2017;14(5):e1002306.

14. Macinko J, Lima-Costa MF. Horizontal equity in health care utilization in Brazil, 1998-2008. Int J Equity Health 2012;11:33.

15. Hudon C. Case management in Primary Care for frequent users of health care services with chronic diseases: a qualitative study of patient and family experience. Ann Fam Med 2015; 13(6):523-528.

16. Lamothe L, Sylvain C, Sit V. Multimorbidity and primary care: Emergence of new forms of network organization. Sante Publique 2015; 27(Supl. 1):129-135.
17. Mendes EV. As redes de atenção à saúde. Brasília: Organização Pan-Americana da Saúde; 2011.

18. Tarrant C, Windridge K, Baker R, Freeman G, Boulton M. 'Falling through gaps': primary care patients' accounts of breakdowns in experienced continuity of care. Family practice 2015; 32(1):82-87.

19. Giovanella L, Mendonça MHM, Almeida PF, Escorel S, Senna MCM, Fausto MCR, Delgado MM, Andrade CLT, Cunha MS, Martins MIC, Teixeira CP. Saúde da família: limites e possibilidades para uma abordagem integral à saúde no Brasil. Cien Saude Colet 2009; 14(3)783-794.

20. Vargas I, Mogollón-Pérez AS, De Paepe P, Ferreira da Silva MR, Unger JP, Vázquez ML. Barriers to healthcare coordination in market based and decentralized public health systems: a qualitative study in healthcare networks of Colombia and Brazil. Health Policy Plan 2016; 31(6):736-748.

21. Bousquat A, Giovanella L, Campos EMS, Almeida PF, Martins CL, Mota PHS, et al. Atenção primária à saúde e coordenação do cuidado nas regiões de saúde: perspectiva de gestores e usuários. Cien Saude Colet 2017; 22(4):1141-1154.

22. Fausto MCR, Campos EMS, Almeida PF, Medina MG, Giovanella L, Bousquat A, Carneiro A, Jerônimo AS, Aleluia IRS, Borges GA, Mota PHS. Itinerários terapêuticos de pacientes com acidente vascular encefálico: fragmentação do cuidado em uma rede regionalizada de saúde. Rev. bras. saúde matern. infant. 2017; 17(Supl. 1):S63-S72

23. De Negri Filho AA. Bases para um debate sobre a reforma hospitalar do SUS: as necessidades sociais e o dimensionamento e tipologia de leitos hospitalares em um contexto de crise de acesso e qualidade [tese]. São Paulo: Universidade de São Paulo; 2016.

24. Organisation for Economic Co-operation and Development (OECD). Health at a Glance 2015: OECD Indicators. Series: Health at a Glance. Paris: OECD Publishing; 2015.

25. Brasil. Portaria n ${ }^{\circ} 3.390$, de 30 de dezembro de 2013. Institui a política Nacional de Atenção Hospitalar (PNHOSP) no âmbito do Sistema Único de Saúde (SUS). Diário Oficial da União 2013; $30 \mathrm{dez.}$

26. Brasil. Ministério da Saúde (MS). Cadernos de Atenção Especializada: Reforma do Sistema da Atenção Hospitalar Brasileira. Brasília: MS; 2004.

27. Uribe Rivera FJ. A démarche estratégica: a metodologia de gestão do Centro Hospitalar Regional Universitário de Lille, França. Cad Saude Publica 1997; 13(1):73-80.

28. Ligress, HCor. Informe do Projeto PROADI SUS 2015 2017 de Plano Diretor de Redes Hospitalares nos Estados do Amazonas, Espírito Santo, Pará e Sergipe. São Paulo: Ligress, HCor; 2017. Mimeo.

Article submitted 05/01/2018

Approved 30/01/2018

Final version submitted 01/03/2018

(cc) Br This is an Open Access article distributed under the terms of the Creative Commons Attribution License 